\title{
Zeitliche und räumliche Skalen der Fluss-Grundwasser-Interaktion: Ein multidimensionaler hydrogeologischer Untersuchungsansatz
}

\author{
Emanuel Huber · Peter Huggenberger • Jannis Epting • Yael Schindler Wildhaber
}

Eingang des Beitrages: 10.7.2012 / Eingang des überarbeiteten Beitrages: 6.11.2012 / Online veröffentlicht: 6.2.2013

(C) Springer-Verlag Berlin Heidelberg 2013

Zusammenfassung Die Prozesse der Fluss-GrundwasserInteraktionen sind stark skalenabhängig und im Allgemeinen stark instationär. Einen für das Prozessverständnis wichtigen Aspekt betreffen die kleinräumigen Strömungsverhältnisse an der Grenzschicht zwischen Oberflächengewässer und Grundwasser im hyporheischen Interstitial. Dies betrifft insbesondere auch Fragestellungen zu Strömungsverhältnissen in Forellenlaichgruben kiesführender Flüsse.

Exemplarisch für kleinere mit Sohlschwellen verbaute und kanalisierte Fließgewässer wurden am voralpinen Schweizer Fluss Enziwigger verschiedene Methoden entwickelt, getestet und kombiniert, die es erlauben die vier Dimensionen (drei räumliche und eine zeitliche) der Interaktion Oberflächengewässer-Interstitialraum-Grundwasser für einzelne Flussabschnitte zu erfassen.

Der Aufbau eines Messnetzes sowie die Durchführung von Feldmessungen lieferten Grundlagen für eine Grundwasserströmungsmodellierung. Kontinuierliche Zeitreihen der Hydraulik, Temperatur und elektrischen Leitfähigkeit im Fließgewässer, an der Gewässersohle sowie im flussnahen

E. Huber · P. Huggenberger · J. Epting ( $\varangle)$

Angewandte und Umweltgeologie,

Departement Umweltwissenschaften, Universität Basel,

Bernoullistraße 32, 4056 Basel, Schweiz

E-Mail: jannis.epting@unibas.ch

E. Huber

E-Mail: emanuel.huber@unibas.ch

P. Huggenberger

E-Mail: peter.huggenberger@unibas.ch

Y. Schindler Wildhaber

Institut für Umweltgeowissenschaften,

Departement Umweltwissenschaften, Universität Basel,

Bernoullistraße 30, 4056 Basel, Schweiz

E-Mail: yael.schindler@unibas.ch
Grundwasser dienten zudem der Identifizierung von Zonen mit signifikantem Fluss-Grundwasser-Austausch und von zeitlich instationären bevorzugten Fließpfaden im Grundwasser bei unterschiedlichen hydrologischen Randbedingungen. Die Resultate der Feldmessungen in Kombination mit der instationären Modellierung und Szenarienentwicklung illustrieren die Bedeutung von sich dynamisch verändernden Infiltrations- und Exfiltrationsmustern im Flussbett.

Spatiotemporal scales of river-groundwater interaction: a multidimensional hydrogeological investigation approach

\begin{abstract}
River-groundwater interactions show strong scale-dependencies and are often strongly transient. In this regard, small-scale flow conditions in the hyporheic zone at the interface between surface- and groundwater can be important for process-understanding. This especially includes questions concerning flow conditions in salmonid redds of gravel-bed rivers. The Swiss subalpine river Enziwigger was chosen as an example for a small channelized river with artificial steps within the riverbed. Several methods were developed, tested and combined that capture the four dimensions (three spatial and one temporal) of the interactions between surface water, the hyporheic zone and groundwater, for individual river segments. The setup of a monitoring network as well as the realization of field-measurements provided data for groundwater flow models. Continuous time series of hydraulic data, temperature and electrical conductivity within the river and the riverbed, as well as within the riverine groundwater, allowed identifying zones with significant exchange of surface water and groundwater. Additionally, the data helped describe the transient character of groundwater flow-paths under various hydrological boundary conditions.
\end{abstract}


Results of the field-measurements in combination with transient groundwater flow modeling and scenario analyses illustrate the relevance of dynamically changing infiltration and exfiltration patterns within the riverbed.

Keywords River-groundwater interaction - Hyporheic flow $\cdot$ Spatiotemporal scales $\cdot$ Redds

\section{Einleitung}

Die Prozesse der Fluss-Grundwasser-Interaktion sind wesentlich durch die Abflussdynamik der Oberflächengewässer geprägt und entsprechend instationär. Die Durchlässigkeiten von Flussbett und Uferzone variieren in Abhängigkeit von Abfluss, Temperatur und Partikeldynamik an der Grenzschicht zwischen Oberflächengewässer und Grundwasser. Zudem machen Heterogenitäten in der Korngrößenzusammensetzung der Flusssohle ein „Upscaling“ der hydraulischen Parameter (Porosität und Permeabilität) über einen bestimmten Abschnitt des Flussbetts schwierig.

Trotz der Bedeutung für die Wasserressourcenbewirtschaftung, die Gewässerökologie und den Grundwasserschutz ist das Verständnis dieser Austauschprozesse ungenügend. Dies betrifft auch die lückenhaften Kenntnisse von Prozessabläufen im hyporheischen Interstitial. Eine besondere Herausforderung sind die verschiedenen Einflüsse auf das Grundwasserfließregime in den unterschiedlichen Maßstabsbereichen, einschließlich der Dynamik der verschiedenen Randzuflüsse sowie der Morphologie und Zusammensetzung des Flussbettes.

Die meisten Feldexperimente bei hohen Abflüssen geben nur bedingt Aufschluss über die effektive Dynamik des Geschiebe- und Partikeltransportes (Ashmore 1988). Experimente, welche die Prozesse untersuchen, die zur Verringerung der Durchlässigkeit der Deckschicht führen, wurden zudem im Labor- und nicht im Feldmaßstab entwickelt (Schälchli 1993, 1995). Dass im Feldmaßstab räumlich komplexe Muster von Veränderungen in der Durchlässigkeit der Flusssohle im Verlauf einzelner Hochwasserereignisse regelmäßig auftreten, zeigen zahlreiche Untersuchungen ( $\mathrm{z}$. B. Doppler et al. 2007, Hoehn \& Cirpka 2006, Naegeli et al. 1995).

Im Rahmen eines interdisziplinären Forschungsprojektes des Schweizer Nationalfonds K-32K1-120486 wurden Methoden entwickelt, um den Einfluss von Feinsedimenten in Flüssen auf die Entwicklung von Forellenlaichgruben in Kiesbänken zu quantifizieren. Primär stand die Frage im Vordergrund, wie die Entwicklung von Fischlaich in den Laichgruben der Kiesbänke jeweils in der Laichperiode von November bis Ende März des darauffolgenden Jahres von der Dynamik dieser Austauschprozesse beeinflusst wird. Von Bedeutung war insbesondere der Einfluss der Sedimentinfiltration in die Poren der Flussbettablagerungen sowie das Erfassen der Instationarität der hydraulischen Verhältnisse, einschließlich der Veränderung von Up- und Downwelling-Zonen (Schindler Wildhaber et al. 2013, in Vorbereitung). Für die Untersuchungen war es notwendig verschiedene Erkundungsmethoden miteinander zu kombinieren, welche es erlauben, Aussagen über die Austauschprozesse (direkte Infiltration) an der wassergesättigten Grenzschicht zwischen Oberflächengewässer und Grundwasser im Maßstab der Laichgruben zu machen.

Mit diesem Beitrag sollen, am Beispiel des verbauten Schweizer Mittelgebirgsflusses Enziwigger und dem regionalen alluvialen Grundwassersystem, die Austauschprozesse Fließgewässer-Interstitialraum-Grundwasser sowie das Grundwasserfließregime in verschiedenen Maßstabsbereichen mit dem Schwerpunkt auf die Dynamik der Strömungsverhältnisse in Forellenlaichgruben charakterisiert werden. Dabei werden verschiedene Betrachtungsmaßstäbe unterschieden (Tab. 1): (A) regional als Teil des Grundwasserkörpers im Tal [100er m bis km]; (B) Bereiche eines Flussabschnittes [10er bis $100 \mathrm{~m}$ ] und (C) lokal im Bereich der Schwellen, Kiesbänke und Forellenlaichgruben [1er bis $10 \mathrm{er} \mathrm{m}]$.

Der Schwerpunkt der Untersuchungen lag auf der Erfassung der zeitlichen und räumlichen Skalen der FlussGrundwasser-Interaktion. Die Haupthypothese der vorliegenden Arbeit ist, dass sich im Bereich von Sohlschwellen lokale Grundwasserfließregime entwickeln, die zu einer Abfolge von Up- und Downwelling-Zonen führen und damit die Strömungsverhältnisse in den Laichgruben beeinflussen. Oberhalb der Flussschwellen infiltriert Flusswasser aufgrund des erhöhten hydraulischen Gradienten. Das infiltrierte Wasser strömt um und unter der Schwelle und exfiltriert unterhalb der Schwelle wieder in das Fließgewässer. Diese lokalen Prozesse werden überlagert von regionalen instationären Prozessen der In- und Exfiltration zwischen dem Fließgewässer und dem regionalen Grundwasserfließregime. Abschließend werden qualitative Aspekte auf der lokalen Skala der untersuchten künstlich angelegten Fischlaichgruben im Zusammenhang mit dem instationären lokalen Grundwasserfließregime diskutiert.

\section{Untersuchungsgebiet}

Die Untersuchungen wurden am kleinen voralpinen Fluss Enziwigger im Schweizer Kanton Luzern im Hügelland südlich von Willisau durchgeführt (Abb. 1). Die Enziwigger entspringt am Nordhang des Napfes auf 1.300 m ü. M. und mündet nach $17 \mathrm{~km}$ in die Wigger. Das gesamte Einzugsgebiet der Enziwigger beträgt $38 \mathrm{~km}^{2}$. Im Haupttal, welches von Süden nach Norden verläuft, wurde die Enziwigger durch Begradigung und Kanalisierung im 20. Jahrhundert stark verkürzt und über weite Strecken auf die östliche 
Tab. 1 Fragen und Ziele für die verschiedenen Untersuchungsskalen sowie Untersuchungsmethoden und Ergebnisse

\begin{tabular}{|c|c|c|c|}
\hline Skale & Fragen/Ziel & Untersuchungsmethode & Ergebnisse \\
\hline $\begin{array}{l}\text { Einzugsgebiet* \& } \\
\text { regionale Grund- } \\
\text { wasserkörper } \\
(100 \mathrm{er} \mathrm{m} \text { bis km) }\end{array}$ & $\begin{array}{l}\text { - Niederschlag-Abflussbildung } \\
\text { - Flächenhaft versickerndes Nieder- } \\
\text { schlagswasser } \\
\text { - Zusammenhang mit regionaler } \\
\text { Grundwasserströmung und Hang- } \\
\text { zustrom }\end{array}$ & $\begin{array}{l}\text { - Auswertung meteorologischer Da- } \\
\text { ten, Grundwasser- und Flusspegel } \\
\text { - Regionales Grundwasserströ- } \\
\text { mungsmodell }\end{array}$ & $\begin{array}{l}\text { - Das Einzugsgebiet hat kaum Spei- } \\
\text { cherkapazität } \\
\text { - Geringe Grundwasserneubildung } \\
\text { aus flächenhaft versickerndem } \\
\text { Niederschlagswasser } \\
\text { - Hangzustrom ist vernachlässigbar, } \\
\text { hat aber einen lokalen Einfluss }\end{array}$ \\
\hline $\begin{array}{l}\text { Flussabschnitt, mehrere } \\
\text { Schwellen } \\
(10 \text { er bis } 100 \mathrm{~m})\end{array}$ & $\begin{array}{l}\text { - Zeitliche und räumliche Charak- } \\
\text { terisierung der Fluss-Grundwasser- } \\
\text { Interaktion }\end{array}$ & $\begin{array}{l}\text { - Auswertung von Grundwasser- und } \\
\text { Flussdaten }\left(\mathrm{P}^{\mathrm{a}}, \mathrm{T}^{\mathrm{b}}, \mathrm{LF}^{\mathrm{c}}\right) \\
\text { - Regionales und lokales Grundwas- } \\
\text { serströmungsmodell }\end{array}$ & $\begin{array}{l}\text { - Auf der regionalen Skale infiltriert } \\
\text { der Fluss in das Grundwasser } \\
\text { - Lokale Grundwasserfließregime } \\
\text { sind komplexer }\end{array}$ \\
\hline $\begin{array}{l}\text { Schwelle, Kiesbänke, } \\
\text { Laichgrube } \\
\text { (1er bis } 10 \text { er m) }\end{array}$ & $\begin{array}{l}\text { - Einfluss Morphologie (Eintiefung } \\
\text { des Flusses, Schwellen, Kolke, To- } \\
\text { pographie Flusssohle) } \\
\text { - Einfluss der Durchlässigkeit der } \\
\text { Flusssohle } \\
\text { - Dynamik In-/Exfiltration } \\
\text { - Erosion/Verringerung der Fluss- } \\
\text { bettdurchlässigkeit } \\
\text { - Definition von Schwellenwerten }\end{array}$ & $\begin{array}{l}\text { - Vermessung Flussbett } \\
\text { - Auswertung von Grundwasser- und } \\
\text { Flussdaten }\left(\mathrm{P}^{\mathrm{a}}, \mathrm{T}^{\mathrm{b}}, \mathrm{LF}^{\mathrm{c}}\right) \\
\text { - Lokales Grundwasserströmungs- } \\
\text { modell } \\
\text { - Analyse Flussbetttemperatur }\end{array}$ & $\begin{array}{l}\text { - Schwelle-Kolk-Reihenfolge prägt } \\
\text { lokale Grundwasserfließregime } \\
\text { stark } \\
\text { - Starke Hochwasserereignisse mit } \\
\text { deutlichen Änderungen der Aus- } \\
\text { tauschprozesse an der Flusssohle }\end{array}$ \\
\hline
\end{tabular}

*Nur Betrachtung Abflussbildung; ${ }^{\text {apegel; }}{ }^{\text {b Temperatur; }}$ c elektrische Leitfähigkeit

Talseite verlegt. Heute sind ca. $60 \%$ der Enziwigger stark beeinträchtigt (Schager \& Peter 2001, 2002). Um die Tiefenerosion der Flusssohle zu verhindern oder wesentlich zu reduzieren wurde die Sohle mit Schwellen stabilisiert und die Böschungen mit Blocksteinen gesichert. Die Sohlschwellen mit einer Metallarmierung wurden alle 10 bis $15 \mathrm{~m}$ in die Flusssohle eingebracht. Dadurch ist das Fließregime hinsichtlich von Strömung und Wassertiefe vergleichsweise monoton. Unterhalb einiger dieser Schwellen haben sich Kolke gebildet, welche lokal die Flusstiefe stark erhöhen. Trotz dieser Flussverbauungen gilt die Enziwigger als gutes Forellenlaichgewässer (Schager \& Peter 2001, 2002). Die Gewässerbettstruktur der Enziwigger ist charakteristisch für viele voralpine Fließgewässer wie auch Mittelgebirgsflüsse und erlaubt es, den Einfluss von wasserbaulichen Maßnahmen, wie z. B. Sohlschwellen, auf die Austauschprozesse der Fluss-Grundwasser-Interaktion in den verschiedenen Maßstabsbereichen zu evaluieren.

Das Untersuchungsgebiet selbst ist ca. $0,1 \mathrm{~km}^{2}$ groß und liegt auf einer mittleren Höhe von ca. $624 \mathrm{~m}$ ü. M. (Abb. 1). Hier ist der Talgrundwasserleiter charakterisiert durch teilweise gering mächtige Quartärbedeckung (bis ca. $13 \mathrm{~m}$ in der Talmitte, Bohrtiefe BP1; Abb. 1d), bestehend aus Bachschuttkegeln, Gehängebildungen, fluvialen Sedimenten hochwürmeiszeitlicher Niederterassenschotter (Landeshydrologie und -geologie 1994) und rezenten Alluvionen. Der Grundwasserstauer ist charakterisiert durch Konglomerate und Sandsteine der oberen Meeres- und oberen Süßwassermolasse.
Die durchschnittliche Jahressumme des Niederschlags im Zeitraum von 2000 bis 2009 beträgt 1055 mm (Messstelle LU 08 Willisau). Hydrologisch ist das Einzugsgebiet durch Schnee im Winter (Dezember bis April) und das Auftreten von starken konvektiven Niederschlägen im Sommer gekennzeichnet. Das Wasserrückhaltevermögen der Böden im Quellgebiet und somit die Speicherkapazität im Einzugsgebiet ist klein. Dadurch ist der Abfluss stark durch Starkniederschlagsereignissen beeinflusst. Der mittlere Abfluss der Enziwigger beträgt ca. $2 \mathrm{~m}^{3} / \mathrm{s}$, der minimale Abfluss liegt bei ca. $1 \mathrm{~m}^{3} / \mathrm{s}$ und der maximale Abfluss bei ca. 10 $\mathrm{m}^{3} / \mathrm{s}$ (Messreihe von Mitte November 2007 bis Mitte November 2008, welche im Rahmen eines Bauprojektes in Willisau aufgezeichnet wurde).

\section{Methoden und Datengrundlagen}

Der multidimensionale Untersuchungsansatz umfasst unterschiedliche Methoden und Skalenbereiche: (1) Erfassen der hydrologischen und Grundlagendaten im Einzugsgebiet der Enziwigger und Darstellung in einem Datenbank-GISSystem; (2) Installation von Messsystemen zur Aufzeichnung hydraulischer und physikalischer Daten im Fließgewässer, in der Gewässersohle und im flussnahen Grundwasser; (3) Aufnahme der Flussbetttopographie sowie (4) Grundwasserströmungsmodellierung und Szenarienberechnungen. 
Abb. 1 Untersuchungen auf verschiedenen Skalen. (a) Lage des Untersuchungsgebiets in der Schweiz. (b) Digitales Höhenmodell (5 m-Auflösung) des Untersuchungsgebiets und Modellperimeter für das regionale Grundwasserströmungsmodell. Dargestellt ist die Verteilung von berechneten Grundwassergleichen (1 m- ̈̈quidistanz) während einer Mittelwassersituation. (c) Digitales Höhenmodel (5 m-Auflösung) und Modellperimeter des lokalen Grundwasserströmungsmodells. Dargestellt sind die Verteilung von berechneten Grundwassergleichen (0,2 m-Äquidistanz) während einer Mittelwassersituation sowie die Lage der Grundwassermessstellen, Sohlschwellen und künstlich angelegten Forellenlaichgruben. (d) 5-fach überhöhtes laterales Höhenprofil durch den Talgrundwasserleiter der Enziwigger, dargestellt mit einem, aus den Bohrtiefen abgeleiteten Schotter-Grundwasserkörper; die Enziwigger wurde über weite Bereiche an den östlichen Talrand verlegt. (e) Darstellung des untersuchten Flussbettabschnittes und Resultate der Flussbettvermessungen zusammen mit der Lage der ufernahen Grundwassermessstellen, Sohlschwellen und künstlich angelegten Forellenlaichgruben
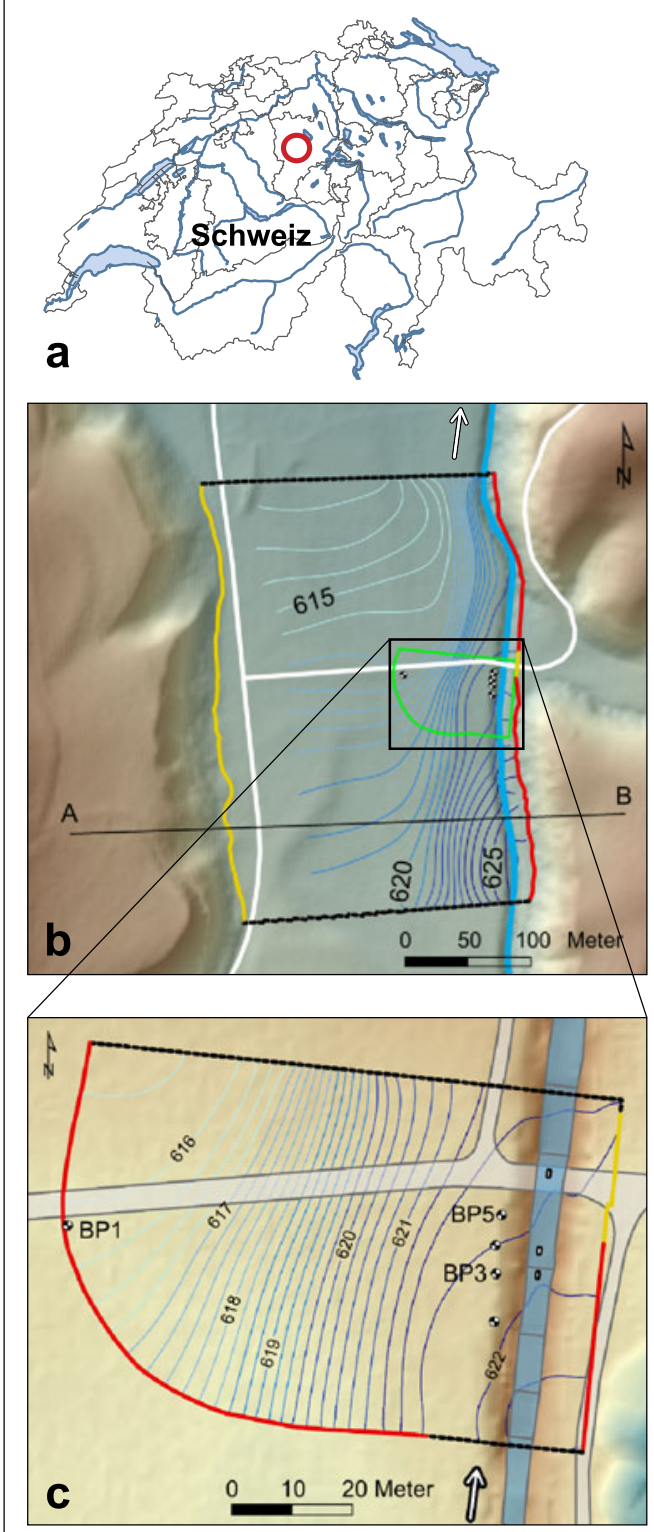
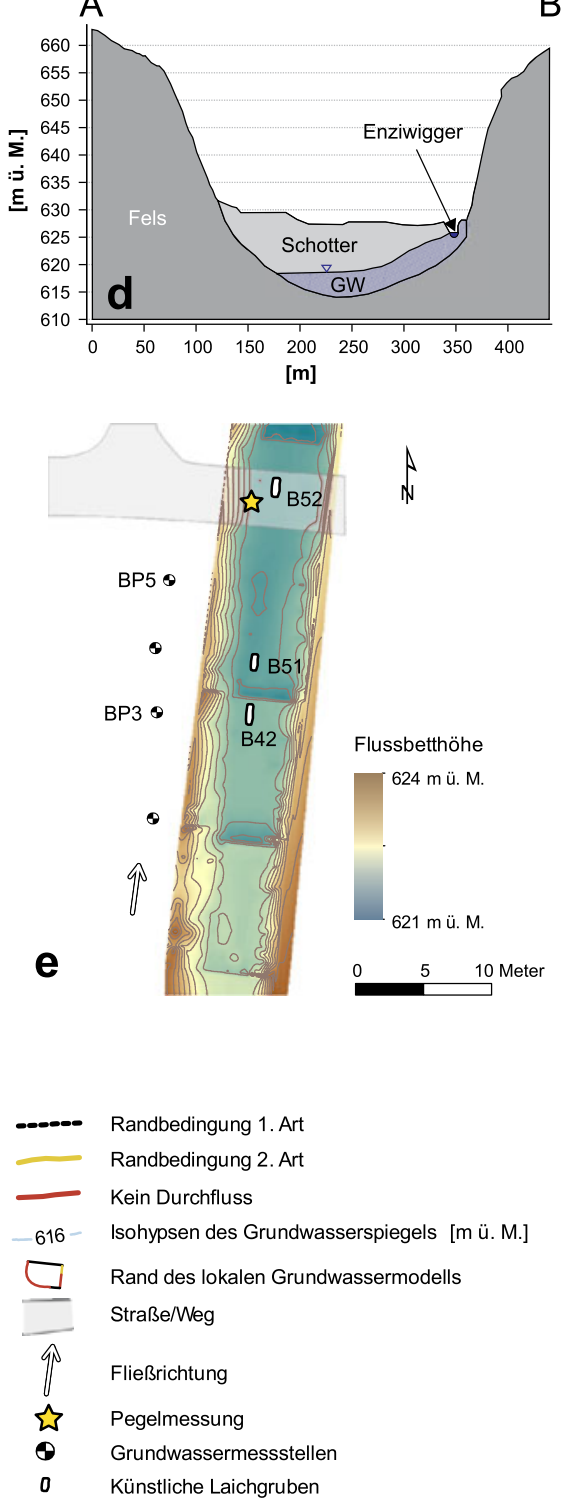

Hydrologische Grundlagen

Die meteorologischen Grundlagendaten basieren auf Messungen der Station Willisau (MeteoSchweiz Stationsnummer 6130). Für die Abschätzung des Beitrags der Schneeschmelze am Gesamtabfluss wurden Lufttemperaturmessungen aus Hergiswil südlich von Willisau (Messintervall 5 min; MeteoSchweiz Stationsnummer 6133) sowie tägliche Schneetiefen aus dem $4 \mathrm{~km}$ entfernten Nebental Luthern (MeteoSchweiz Stationsnummer 6630) verwendet.

Messsysteme zur Aufzeichnung hydraulischer und physikalischer Parameter

Mit der Direct-Push-Technik (Geoprobe Kansas/USA) wurden für Standort B insgesamt 5 Grundwassermessstel- len im Talbereich und am Flussufer errichtet (Sondiergröße von 2,152“ für den Ausbau von 1"“-MessstellenRammsondierungen, Abb. 1). Die Sondierungen konnten bis zur Felsoberfläche (Annahme) in Tiefen von ca. 12,8 $\mathrm{m}$ im Talbereich und von ca. 4,4 $\mathrm{m}$ am Flussufer abgeteuft werden. Die Sondierung in der Talmitte (BP1) sowie zwei Sondierungen am Flussufer (BP3, BP5) sind als Grundwassermessstellen ausgebaut und mit Messsonden zur kontinuierlichen Aufzeichnung des Grundwasserpegels, der Grundwassertemperatur und der elektrischen Leitfähigkeit (nur BP3 und BP5) ausgestattet. Während mit den Messungen im Uferbereich die lokalen Austauschprozesse im Bereich der Flussabschnitte untersucht wurden, diente die Messung in der Talmitte der Abschätzung des Einflusses des regionalen auf das lokale Grundwasserfließregime. Die Analyse 
von Fluss- und Grundwasserpegeldaten im Uferbereich und in der Talmitte ermöglichten eine erste Abschätzung der Inund Exfiltrationsverhältnisse.

Für die Untersuchungen in der Gewässersohle wurde die Temperatur in zwei unterschiedlichen Tiefen (zwischen 10 und $38 \mathrm{~cm}$ ) in drei künstlich angelegten Laichgruben kontinuierlich gemessen (Abb. 1). Die tägliche Temperaturamplitudendifferenz zwischen dem Oberflächen- und Interstitialwasser wurde verwendet um die spezifische Austauschrate $q$ zu berechnen (Hatch et al. 2006, Keery et al. 2007). Eine hohe spezifische Austauschrate führte zu einer Annäherung der Temperaturverläufe in den zwei Tiefen an den Temperaturverlauf des Oberflächenwassers, eine niedrige Austauschrate zu einem abgeflachten Verlauf in der Tiefe. Exfiltrierendes Grundwasser konnte bei einer zusätzlich verstärkten Abflachung ermittelt werden. Diese Methode erlaubte es, zwei $q$-Werte pro Tag zu berechnen. Somit konnte lokal der zeitliche Verlauf der Durchlässigkeiten nach dem Bau der Laichgruben im November bis zum Schlupf der Eier im März beschrieben werden. Die berechneten Durchlässigkeitswerte der Monate Februar und März wurden gemittelt, in der Annahme, dass sich bis dahin die anfänglich erhöhte Durchlässigkeit durch den Bau der Laichgrube auf einem natürlichen Level stabilisiert hat.

\section{Flussbetttopographie}

Da Änderungen der Struktur und Zusammensetzung der Flusssohle nach starken Hochwässern mehrmals festgestellt wurden, fanden insgesamt drei Neuvermessungen der Flussbetttopographie statt (Abb. 1e). Differenzenkarten von aufeinanderfolgenden Messungen ermöglichten es, Bereiche in den untersuchten Flussabschnitten auszuweisen, in denen Akkumulations- oder Erosionsprozesse während der Hochwasserereignisse dominiert haben.

Grundwasserströmungsmodell und Szenarienberechnungen

Die Grundwasserströmungsberechnungen wurden in einem regionalen und lokalen Maßstab durchgeführt und dienen der Abschätzung von räumlichen und zeitlichen Variationen des Grundwasserfließregimes (Grundwasserfließfeld, -fließgeschwindigkeiten und Wasserbilanzen). Mit den Modellen wurden verschiedene Prozesse detailliert untersucht, einschließlich der Sensitivität von Randbedingungen sowie des zeitlich und räumlich instationären Charakters der Fluss-Grundwasser-Interaktion. Ein Schwerpunkt liegt auf der Beantwortung der Frage nach dem Zusammenhang zwischen Oberflächenmorphologie des Fließgewässers und der Wasserwegsamkeit im hyporheischen Interstitial. Eine Abschätzung der Einflüsse von verschiedenen Parametern und Randbedingungen (z. B. Flussbettmorphologie, Verteilung der Flussbettdurchlässigkeit), beruht auf Szenarienberechnungen mit dem kalibrierten Grundwassermodell.
Die Grundwasserströmungsberechnungen erfolgten mit GMS (Groundwater Modeling System 7.1; Environmental Modeling Systems 2002) auf der Basis von MODFLOW (McDonald \& Harbaugh 1996). Die Randbedingungen des regionalen Modells $(230 \mathrm{~m} \times 340 \mathrm{~m}$, Auflösung $2 \mathrm{~m} \times 2 \mathrm{~m}$ ) wurden auf die Randbedingungen des lokalen Modells $(110 \mathrm{~m} \times 60 \mathrm{~m}$, Auflösung $0,5 \mathrm{~m} \times 0,5 \mathrm{~m})$ übertragen (Abb. 1). Für die Beschreibung des Verlaufs der Felsoberfläche wurden Angaben zu Eindringtiefen der DirectPush-Erkundung herangezogen. Für das regionale Modell wurde weiterhin angenommen, dass im Bereich der westlichen und östlichen Hangbereiche die Hangsteigung im Zusammenhang mit dem Verlauf des Grundwasserstauers steht (Abb. 1b-1d). Für das lokale Modell wurden zudem die hochaufgelösten Flussbettvermessungen berücksichtigt (Abb. 1e). Die Randbedingungen für die Grundwasserströmungsmodellierung werden in Tab. 2 zusammengefasst (Abb. 1). Aufbauend auf flusssedimentologischen Überlegungen und dem Vorhandensein von Grundwassermessungen wurden die Durchlässigkeiten des Grundwasserleiters und an der Flusssohle instationär (220 Tage, Tagesauflösung) invers kalibriert (Pilot Point, PEST; Doherty 1994) sowie eine Sensitivitätsanalyse der relevanten Parameter durchgeführt. Kalibrierte Durchlässigkeiten des Grundwasserleiters bewegen sich zwischen $10^{-2}$ und $10^{-4} \mathrm{~m} / \mathrm{s}$. Im Bereich des Hauptgrundwasserstroms und im unmittelbaren Bereich der Enziwigger sind die kalibrierten Durchlässigkeiten tendenziell höher. In Abb. 3 sind die kalibrierten Durchlässigkeiten (19 bis 62 1/d) der Flusssohle für die verschiedenen Gewässerabschnitte dargestellt.

Mit dem kalibrierten lokalen Grundwasserströmungsmodell wurden anschließend Szenarien definiert. Zusätzlich zum Ausgangsmodell (Szenario 1) wurde ein Modellszenario nur mit den Sohlschwellen und ohne differenzierte Flussbetttopographie (Szenario 2) sowie ein Modellszenario mit einem gleichmäßigen Gefälle ohne Sohlschwellen und Flussbetttopographie (polynomial ( $\mathrm{Grad} 2)$ interpoliert, Szenario 3) vorbereitet. Die Szenarienberechnungen erlauben es, die Austauschraten und Fließgeschwindigkeiten zwischen dem Modell mit hochaufgelöster Flussbetttopographie mit den Modellszenarien mit monotonerer Flussbetttopographie zu vergleichen.

\section{Resultate}

Hydrologie

Abbildung 2 zeigt die Zeitreihen der Tagessummen des Niederschlags, der Schneetiefe, der Lufttemperatur im Einzugsgebiet der Enziwigger und den Verlauf des Flusspegels für den Zeitraum von November 2010 bis Ende März 
Tab. 2 Definition Modellrandbedingungen (Abb. 1)

\begin{tabular}{|c|c|c|}
\hline Randbedingung & Art & Beschreibung \\
\hline $\begin{array}{l}\text { regionale Grundwasserflüsse } \\
\text { in das Modellgebiet hinein } \\
\text { (im Süden) und hinaus (im } \\
\text { Norden) }\end{array}$ & $\begin{array}{l}\text { 1. Art (Festpotenzial, variabel über die } \\
\text { Zeit) }\end{array}$ & $\begin{array}{l}\text { Regionales Modell: Lineare Interpolation von Piezometerhöhen } \\
\text { zwischen zwei im Haupttal liegenden Grundwasserpegeln }\end{array}$ \\
\hline $\begin{array}{l}\text { Seitenzuflüsse aus den } \\
\text { Hangbereichen }\end{array}$ & $\begin{array}{l}\text { 2. Art (Randzufluss und -Abfluss, } \\
\text { variabel über die Zeit) }\end{array}$ & $\begin{array}{l}\text { Diese Zuflüsse entsprechen der Grundwasserneubildung } \\
\text { (meteorologische Daten, siehe Sektion Hydrologische Grundlagen) } \\
\text { integriert auf die Fläche der Hangeinzugsgebiete, welche mittels } \\
\text { hydrologischer Oberflächenmodellierung auf Basis des digitalen } \\
\text { Höhenmodells evaluiert wurden }\end{array}$ \\
\hline Enziwigger & $\begin{array}{l}\text { 3. Art (halbdurchlässiger Rand, variable } \\
\text { Abflusstiefe über die Zeit und } \\
\text { Austauschrate) }\end{array}$ & $\begin{array}{l}\text { - Regionales Modell: Linie mit „River“-Package } \\
\text { - Lokales Modell: flächig als „General Head Boundary“ (GHB); } \\
\text { - Austauschrate (Conductance-Wert): kalibriert }\end{array}$ \\
\hline $\begin{array}{l}\text { Grundwasserneubildung } \\
\text { durch flächig versickerndes } \\
\text { Niederschlagswasser }\end{array}$ & & $\begin{array}{l}\text { Tägliche Niederschläge, einschließlich Berücksichtigung von } \\
\text { Schneehöhen, Lufttemperatur und Jahreszeiten. Die Abschätzung des } \\
\text { Beitrags der Schneeschmelze basiert auf einem einfachen } \\
\text { Temperaturindex-Modell: Wenn die Summe der stündlich } \\
\text { gemessenen Lufttemperatur negativ ist, werden die Niederschläge als } \\
\text { Schnee betrachtet. Sobald diese Summe positiv ist, wird der } \\
\text { Schneeschmelzvorrat proportional zur Temperatursumme und zu } \\
\text { einem Schneeschmelzfaktor von } 0,01\left(\text { Tag } \times{ }^{\circ} \mathrm{C}\right)^{-1} \text { verringert } \\
\text { (Debele \& Srinivasan 2005). Das Wasservolumen von } \\
\text { Niederschlägen bei Temperaturen über } 0{ }^{\circ} \mathrm{C} \text { trägt mit einem Faktor } \\
\text { von } 0,3 \text { zur Grundwasserneubildung bei. }\end{array}$ \\
\hline
\end{tabular}

2011. Deutlich ist ein Zusammenhang von einzelnen Niederschlagsereignissen und dem Flusspegellauf in der Enziwigger und zwischen Schneetiefe und Temperaturverlauf zu beobachten. Temperaturanstiege bei einer vorhandenen Schneedecke führen zu Schmelzereignissen, welche durch die Erhöhung des Oberflächenabflusses zu einem Anstieg des Flusspegels führen. Sowohl bei Niederschlag- als auch auf Schneeschmelzereignissen steigt der Flusspegel rasch an. Dies ist ein Hinweis für eine kleine Speicherkapazität des Molasseuntergrundes im Einzugsgebiet ohne wesentliche Pufferwirkung des Grundwassers.

Hydraulische und physikalische Parameter

Tabelle 3 fasst die wesentlichen Resultate der Messungen im Fluss, im Grundwasser und in den Laichgruben zusammen. Die Untersuchungen orientierten sich vor allem an den Laichperioden 2009/2010 und 2010/2011. Messungen des Flusspegels erstreckten sich für die erste Laichperiode über den Zeitraum von November 2009 bis August 2010 (teilweise blieben einzelne Messgeräte im Fluss länger installiert) und für die zweite Laichperiode über den Zeitraum von November 2010 bis Ende März 2011. Die Messungen in den Grundwasserpegeln lieferten kontinuierliche Zeitreihen über den gesamten Zeitraum vom November/Dezember 2009 bis Dezember 2010. Die im Rahmen dieser Arbeit diskutierten Austauschprozesse im Skalenbereich der Laichgruben konzentrieren sich auf die erste Laichperiode und den Zeitraum vom November bis Ende März 2010.
Der Grundwasserpegel in der Messstelle BP1 im Tal (Abb. 1) zeigt ein gedämpftes Signal des Pegelverlaufs. Ein Minimum des Grundwasserpegels kann zwischen Februar und März 2010 beobachtet werden, welches darauf zurückgeführt werden kann, dass in diesem Zeitraum wenige Hochwasserereignisse stattfanden. Generell ist der Grundwasserpegel im Vergleich zum Flusspegel über $5 \mathrm{~m}$ niedriger. Auf der regionalen Skala dominiert somit der Gradient vom Fluss zum Grundwasser und der Prozess der Flusswasserinfiltration (Abb. 1, 2 und Tab. 1, 3). Auch die Temperaturdaten in der Grundwassermessstelle BP1 im Tal zeigen ein gedämpftes Signal im Jahresverlauf und eine saisonale Verzögerung im Vergleich zum Verlauf der Lufttemperatur von ca. 124 Tagen. Diese Retardation resultiert in einem im Vergleich zum Verlauf der Lufttemperatur verzögerten Auftreten von Extremwerten. Temperaturmaxima werden im Dezember und Temperaturminima im Juni beobachtet.

Abbildung 2 zeigt auch den Verlauf der Grundwassermessungen in den beiden Messstellen am Flussufer BP3 und BP5 im Vergleich zu den Messungen im Fluss und im Talgrundwasserleiter (Abb. 1 und Tab. 3). Lokal im Bereich von Messstelle BP3 oberhalb einer Sohlschwelle dominieren flusswasserinfiltrierende und im Bereich von Messstelle BP5 unterhalb einer Sohlschwelle exfiltrierende Verhältnisse. Die Grundwasserpegeldaten in den Grundwassermessstellen am Flussufer verlaufen ähnlich wie der Flusspegel. Im Frühling ist in Grundwassermessstelle BP5, 


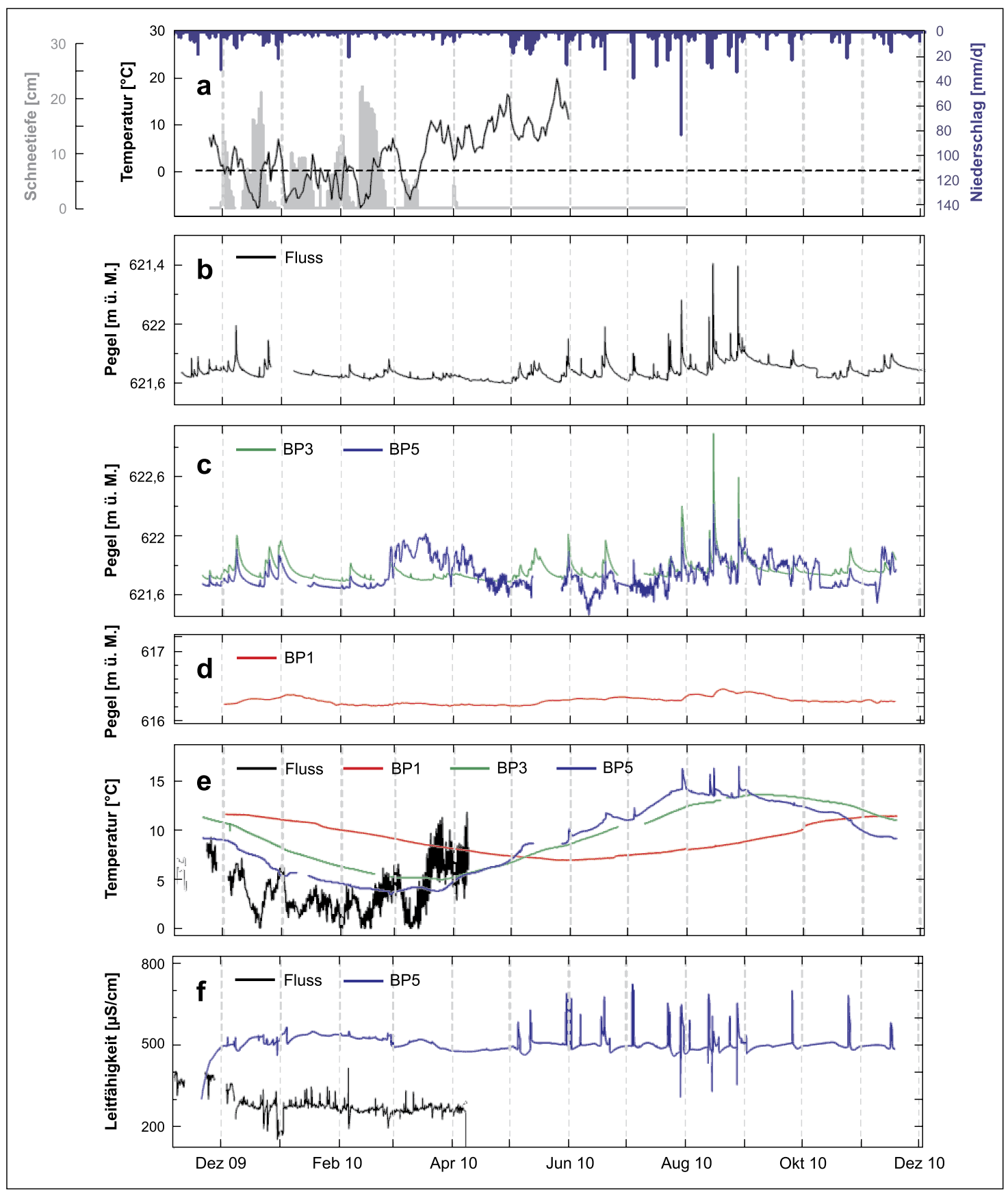

Abb. 2 Zeitreihen von: (a) meteorologischen Daten im Einzugsgebiet der Enziwigger; (b) Pegeldaten im Fluss; (c) Pegeldaten im ufernahen Grundwasser (BP3 und 5, siehe Abb. 1); (d) Pegeldaten im Talgrundwasserleiter (BP1, siehe Abb. 1); (e) Temperaturdaten im Fluss und im
Grundwasser (BP1, 3 und 5, siehe Abb. 1) und (f) Daten der elektrischen Leitfähigkeiten im Fluss und im Grundwasserpegel BP5 (BP5, siehe Abb. 1) welche ansonsten einen gleichen Pegelverlauf wie BP3 zeigt, ein abweichender Pegelverlauf erkennbar. Diese Abweichung wird auf den Einfluss des Hangzustroms nach Schneeschmelzereignissen zurückgeführt (Abb. 2). Saisonale Verzögerungen der Grundwassertemperaturen im Vergleich zum Verlauf der Lufttemperatur liegen bei 29 bis 50 Tagen. Generell haben die Grundwassertemperaturen eine kleinere Amplitude als der Fluss und das Signal ist verzögert (Abb. 2). Tägliche Temperaturschwankungen im Fluss können im flussnahen Grundwasser nicht mehr beobachtet werden. Temperaturmessungen in Grundwassermessstelle BP5 unterhalb einer Sohlschwelle zeigen eine stärkere Reaktion auf Temperaturschwankungen des Flusswassers als jene in BP3 oberhalb der Schwelle in der eine größere Dämpfung 
Tab. 3 Zusammenfassung der Resultate der Fluss- und Grundwassermessungen (BP1, 3 und 5, siehe Abb. 1 und 2), der Temperaturanalysen in den Forellenlaichgruben (B42, 51 und 52, siehe Abb. 1) und der Grundwasserströmungsmodellierung

\begin{tabular}{|c|c|c|c|c|c|c|c|}
\hline & \multirow[t]{2}{*}{$\begin{array}{l}\text { Messzeitraum } \\
(\text { MM.YYYY) }\end{array}$} & \multirow{2}{*}{$\begin{array}{l}\text { Mittlerer Pegel } \\
\text { (m ü. M.) } \pm \\
\text { Standard- } \\
\text { Abweichung } \\
{[\mathrm{m}]}\end{array}$} & \multirow{2}{*}{$\begin{array}{l}\text { Mittlere } \\
\text { Temperatur } \pm \\
\text { Standard- } \\
\text { Abweichung } \\
{\left[{ }^{\circ} \mathrm{C}\right]} \\
\end{array}$} & \multirow{2}{*}{$\begin{array}{l}\text { Saisonale } \\
\text { Temperatur- } \\
\text { amplitude } \\
\alpha\left[{ }^{\circ} \mathrm{C}\right]\end{array}$} & \multirow{2}{*}{$\begin{array}{l}\text { Saisonale } \\
\text { Verzögerung im } \\
\text { Vergleich zum } \\
\text { Flusssignal } \tau_{T} \\
\text { [d] } \\
\end{array}$} & \multicolumn{2}{|c|}{$\begin{array}{l}\text { Berechnete spezifische Austauschraten } \pm \\
\text { Standard-Abweichung }\left[\mathrm{m} \mathrm{d}^{-1}\right]\end{array}$} \\
\hline & & & & & & Temperaturanalyse & Grundwassermodell \\
\hline Fluss & $\begin{array}{l}11.2009-08.2010 \\
11.2010-03.2011\end{array}$ & $621,66 \pm 0,05$ & 9,47 & 7,07 & - & - & - \\
\hline BP1 & $12.2009-04.2011$ & $616,28 \pm 0,05$ & 9,15 & 2,2 & 124,1 & - & - \\
\hline BP3 & $11.2009-04.2011$ & $621,75 \pm 0,07$ & 9,7 & 4,2 & 47,97 & - & - \\
\hline BP5 & 11.2009-03.2011 & $621,72 \pm 0,09$ & 9,25 & 5,14 & 29,47 & - & - \\
\hline B42 & $02.2010-03.2010$ & - & $3,32 \pm 2,16$ & - & - & $0,13 \pm 0,01$ & $1,35 \pm 0,02$ \\
\hline B51 & $02.2010-03.2010$ & - & $3,19 \pm 2,11$ & - & - & $0,11 \pm 0,02$ & $-0,24 \pm 0,04$ \\
\hline B52 & $02.2010-03.2010$ & - & $3,39 \pm 2,09$ & - & - & $0,12 \pm 0,01$ & $0,59 \pm 0,06$ \\
\hline
\end{tabular}

und Verzögerung von Temperatursignalen aus dem Fluss beobachtet werden kann (eine Interpretation für diese Beobachtung wäre, dass nur bei vergleichsweise großen Hochwasserereignissen eine direkte Infiltration von Flusswasser stattfindet).

Die elektrischen Leitfähigkeiten wurden nur im Fluss und in der Grundwassermessstelle BP5 gemessen. Die mittlere elektrische Leitfähigkeit im Fluss liegt bei $276 \mu \mathrm{S} / \mathrm{cm}$ und bei Grundwassermessstelle BP5 bei $504 \mu \mathrm{S} / \mathrm{cm}$. Während bei kleineren und mittleren Hochwasserereignissen ein Ansteigen der elektrischen Leitfähigkeit beobachtet werden kann, kann bei großen Hochwasserereignissen ein Abfallen der elektrischen Leitfähigkeit beobachtet werden (Eine Interpretation für diese Beobachtung wäre, dass während kleinerer und mittlerer Hochwasserereignisse Niederschlagwasser lokal über die ungesättigte Zone und bevorzugte Fließpfade (Makroporen) versickert. Da das Gebiet landwirtschaftlich genutzt wird, führt der Transport von nitrathaltigem Wasser über die ungesättigte Zone zu den Anstiegen der elektrischen Leitfähigkeit. Bei großen Hochwasserereignissen hingegen führt die Infiltration von Flusswasser mit niedrigen elektrischen Leitfähigkeiten zu einem $\mathrm{Ab}$ fallen der elektrischen Leitfähigkeit im ufernahen Grundwasser).

Die Resultate der Temperaturmessungen in den künstlichen Forellenlaichgruben (Abb. 1) werden in Schindler Wildhaber et al. (in Vorbereitung) detailliert beschrieben. In Tab. 3 werden die für die vorliegende Arbeit relevanten wesentlichen Resultate zusammengefasst. Mittlere Temperaturen im Flussbett für Messstandort B42 liegen bei $3,32{ }^{\circ} \mathrm{C}$, für Messstandort B51 bei $3,19^{\circ} \mathrm{C}$ und für Messstandort B52 bei $3,39^{\circ} \mathrm{C}$. Berechnete Austauschraten durch das Flussbett liegen für Messstandort B42 bei $0,13 \mathrm{~m} / \mathrm{d}$, für Messstandort B51 bei $0,11 \mathrm{~m} / \mathrm{d}$ und für Messstandort B52 bei 0,12 $\mathrm{m} / \mathrm{d}$.
Lokales und regionales Grundwasserströmungsmodell

Die Quantifizierung des zeitlichen Verlaufs der FlussGrundwasser-Interaktion erfolgt mithilfe regionaler und lokaler hochauflösender kalibrierter Grundwasserströmungsmodelle. Abbildung 1 zeigt das berechnete Grundwasserfließregime für das regionale und lokale Grundwasserströmungsmodell für eine Mittelwassersituation am 20. April 2010. Das berechnete Grundwasserfließregime für den regionalen Maßstabsbereich bildet vor allem die regionale Grundwasserströmung im Talgrundwasserleiter von Süden nach Norden sowie den Einfluss der Flusswasserinfiltration im ufernahen Bereich nach. Deutlich ist der hydraulische Gradient vom Fließgewässer zum Talgrundwasserleiter zu erkennen. Das berechnete Grundwasserfließregime für den lokalen Maßstabsbereich bildet vor allem den Einfluss der Flusswasserinfiltration im ufernahen Bereich und den Übergang zur regionalen Grundwasserströmung nach.

Die berechneten Wasserbilanzen für das regionale und lokale Grundwasserströmungsmodell lassen sich wie folgt zusammenfassen. Im regionalen Maßstabsbereich können 70 $\%$ der Gesamtwasserbilanz der Flusswasserinfiltration und $30 \%$ dem Talgrundwasserzustrom aus dem Süden (inflow) sowie $12 \%$ der Gesamtwasserbilanz der Grundwasserexfiltration und $88 \%$ dem Talgrundwasserabstrom gegen Norden (outflow) zugeschrieben werden. Im lokalen Maßstabsbereich können $86 \%$ der Gesamtwasserbilanz der Flusswasserinfiltration, $12 \%$ dem Talgrundwasserzustrom aus dem Süden und $2 \%$ dem Hangwasserzustrom (inflow) sowie $30 \%$ der Gesamtwasserbilanz der Grundwasserexfiltration und $70 \%$ dem Talgrundwasserabstrom gegen Norden (outflow) zugeschrieben werden. Die Zahlen verdeutlichen die Abhängigkeit der Gesamtwasserbilanzen vom betrachteten Maßstab. Im regionalen Maßstab machen In- und Exfiltration von Fluss- und Grundwasser $70 \%$ und $12 \%$ im lokalen Maßstab hingegen $86 \%$ und $30 \%$ der Gesamtwasserbilanz aus. 
Tabelle 4 fasst die berechneten Aufenthaltszeiten sowie die Länge der Fließpfade von Flussinfiltrat zwischen der

Tab. 4 Berechnete Aufenthaltszeiten und Fließpfade zu den Grundwassermessstellen (BP1, 3 und 5, siehe Abb. 1) für die regionale und lokale Grundwasserströmungsmodellierung und die verschiedenen Szenarienberechnungen

\begin{tabular}{lllll}
\hline & $\begin{array}{l}\text { Regionales } \\
\text { Modell }\end{array}$ & \multicolumn{2}{l}{ Lokale Modelle } \\
\cline { 3 - 5 } & Szenario 1 & Szenario 2 & Szenario 3 \\
\hline BP1 & & & \\
$t(\mathrm{~d})$ & $12,3 \pm 0,4$ & $14,2 \pm 0,5$ & $14,1 \pm 0,5$ & $14,1 \pm 0,5$ \\
$d(\mathrm{~m})$ & $92,6 \pm 1,7$ & $90,4 \pm 0,7$ & $89,4 \pm 0,8$ & $89,6 \pm 0,9$ \\
BP3 & & & & \\
$t(\mathrm{~d})$ & $0,5 \pm 0,2$ & $4,3 \pm 1,2$ & $4,5 \pm 1,4$ & $3,2 \pm 0,5$ \\
$d(\mathrm{~m})$ & $9,5 \pm 2,8$ & $21,3 \pm 3,8$ & $20,2 \pm 3,3$ & $21,9 \pm 1,8$ \\
BP5 & & & & \\
$t(\mathrm{~d})$ & $0,8 \pm 0,2$ & $4,2 \pm 3,2$ & $4,3 \pm 3,2$ & $3,3 \pm 1,7$ \\
$d(\mathrm{~m})$ & $11,6 \pm 2,0$ & $13,3 \pm 0,5$ & $13,3 \pm 0,5$ & $23,6 \pm 4,8$ \\
\hline
\end{tabular}

Enziwigger und den verschiedenen Grundwassermessstellen zusammen. Infiltriertes Flusswasser benötigt demnach für einen Fließpfad von ca. $93 \mathrm{~m}$ ca. 12 Tage um Grundwassermessstelle BP1 im Tal zu erreichen. Hingegen erreicht infiltriertes Flusswasser die ufernahen Grundwassermessstellen auf Fließpfaden von ca. 10 und $12 \mathrm{~m}$ schon nach ca. 0,5 und 0,8 Tagen.

Abbildung 3 zeigt die räumliche Verteilung der berechneten Flussinfiltration und Grundwasserexfiltration im Flussbett des untersuchten Gewässerabschnittes für verschiedene Wasserstände. Deutlich ist, unabhängig vom Wasserstand, die Dominanz der Infiltration oberhalb und der Exfiltration unterhalb der Sohlschwellen. Vor den Schwellen erreicht die Infiltration von Flusswasser in das Grundwasser Werte zwischen 2 und $3,5 \mathrm{~m} / \mathrm{d}$, die Exfiltration von Grundwasser in den Fluss unterhalb erreicht Werte zwischen 2 und $3,5 \mathrm{~m} / \mathrm{d}$. Im Bereich zwischen den Schwellen dominiert die Infiltration von Flusswasser in das Grundwasser, was auch durch das regionale Grundwasserfließregime und dem hohen Gradienten vom Fluss zum Talgrundwasserlei-

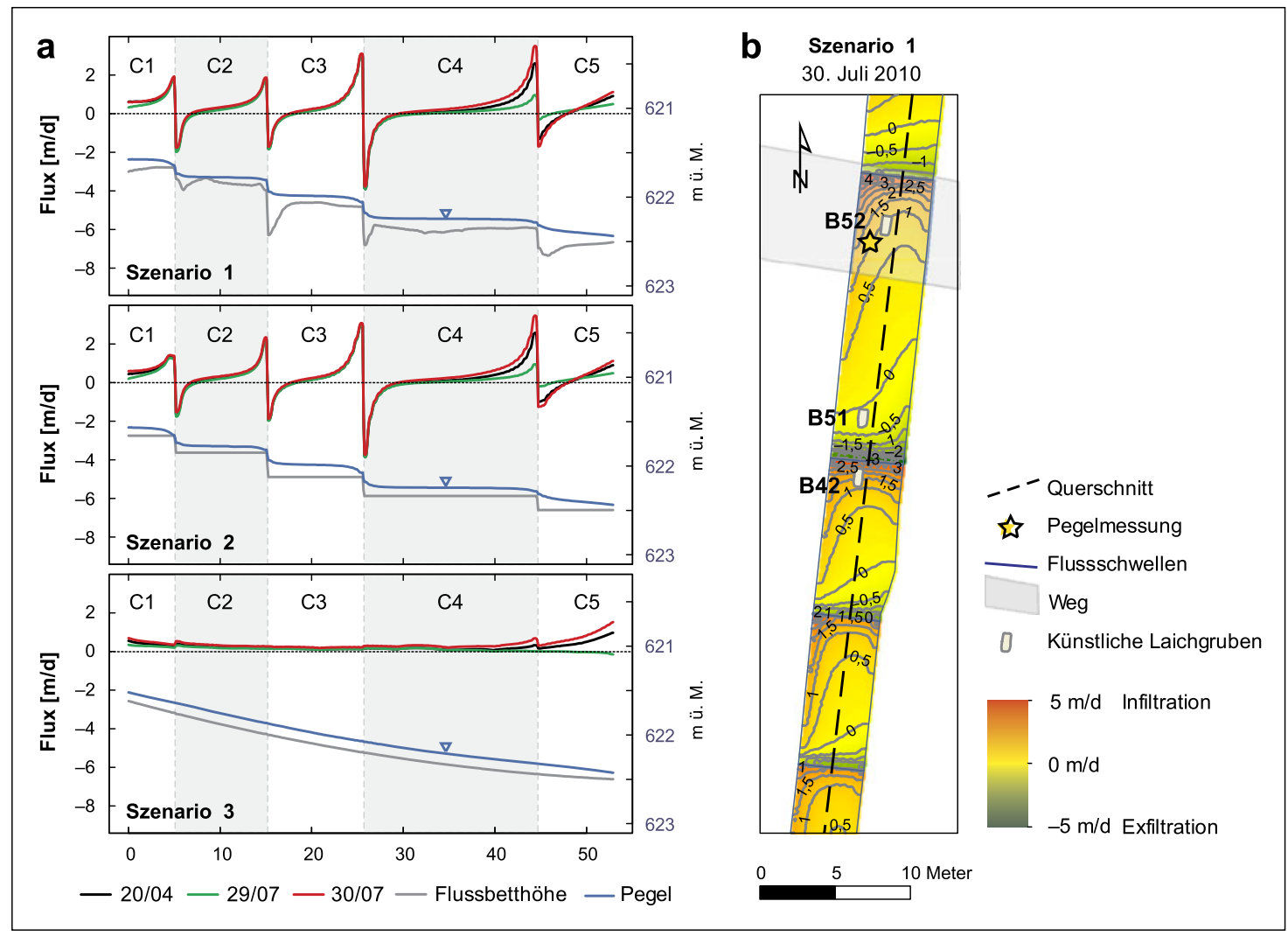

Abb. 3 (a) Räumliche Verteilung der Austauschraten im Flussbett des untersuchten Gewässerabschnitts der Enziwigger für drei Zeitpunkte für das lokale Grundwasserströmungsmodell (Szenario 1). Der 20. April 2010 sowie der 29. Juli 2010 entsprechen einem niedrigen Wasserstand, während des 30. Juli 2010 fand ein Hochwasserereignis statt (Abb. 2). Auch dargestellt sind der Flusspegel und der Verlauf des Flussbettes in der Mitte der Enziwigger. Kalibrierte Durchlässig- keit der Flusssohle: $\mathrm{C} 1=19\left(\mathrm{~m}^{2} / \mathrm{d}\right) / \mathrm{m}^{2}, \mathrm{C} 2=50\left(\mathrm{~m}^{2} / \mathrm{d}\right) / \mathrm{m}^{2}, \mathrm{C} 3=42$ $\left(\mathrm{m}^{2} / \mathrm{d}\right) / \mathrm{m}^{2}, \mathrm{C} 4=62\left(\mathrm{~m}^{2} / \mathrm{d}\right) / \mathrm{m}^{2}, \mathrm{C} 5=19\left(\mathrm{~m}^{2} / \mathrm{d}\right) / \mathrm{m}^{2}$. (b) Berechnete Austauschraten für Szenario 1 während Hochwasser (Abb. 2). Positive Werte entsprechen einer Infiltration vom Flusswasser in das hyporheische Interstitial; negative Werte entsprechen einer Exfiltration vom Grundwasser in den Fluss 


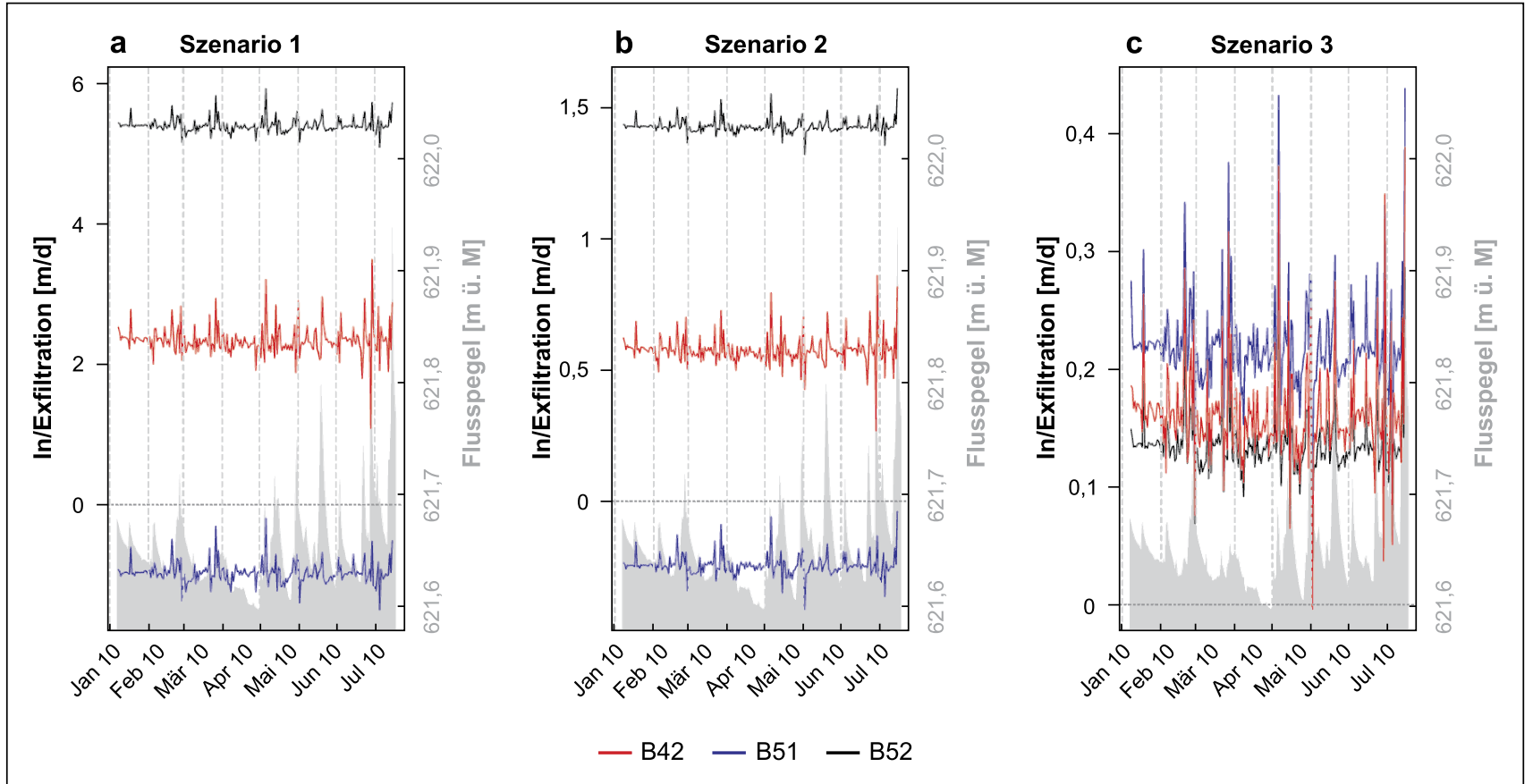

Abb. 4 Zeitliche Verläufe der berechneten Austauschraten durch die künstlich angelegten Forellenlaichgruben (B42, 51 und 52, siehe Abb. 1) für den gesamten instationär modellierten Zeitraum. Positive Werte entsprechen einer Infiltration vom Flusswasser in das hypor- heische Interstitial; negative Werte entsprechen einer Exfiltration vom Grundwasser in den Fluss. Auch dargestellt ist der Verlauf des Flusspegels in der Enziwigger

Flussbett zu berechnen, sind die spezifischen Austauschraten, welche aus der Temperaturmethode abgeleitet wurden, nur für die lokale Skala der Forellenlaichgrube charakteristisch (dm-Bereich).

Szenarienberechnungen mit dem lokalen Grundwasserströmungsmodell

Zusätzlich zum Ausgangsmodell (Szenario 1) wurden mit dem kalibrierten lokalen Grundwasserströmungsmodell Szenarien definiert. Ein Szenario (Szenario 2) berücksichtigt nur die Sohlschwellen nicht aber die differenzierte Flussbetttopographie (Abb. 3b, 5). Ein weiteres Szenario (Szenario 3) berücksichtigt lediglich ein gleichmäßiges Gefälle ohne Sohlschwellen und Flussbetttopographie (polynomial (Grad 2) interpoliert, Abb. 3).

Abbildung 3a zeigt den Verlauf des Flussbettes in der Mitte der Enziwigger für die drei Szenarien sowie berechnete Austauschraten für den gesamten simulierten Gewässerabschnitt. In Szenarien 1 und 2 ist zu erkennen, dass vor den Sohlschwellen die Infiltration von Flusswasser in das hyporheische Interstitial und hinter der Sohlschwelle die Exfiltration von Grundwasser in das Flusswasser dominiert. Bezüglich der Verteilung von In- und Exfiltrationsraten ist der Unterschied zwischen den Szenarien 1 und 2 gering. Die Exfiltration von Grundwasser in den Fluss unterhalb der Sohlschwellen nimmt wegen der fehlenden Kolke für Szenario 
Abb. 5 Strömungsvektoren und Zuströmbereiche zu den Grundwassermessstellen sowie Räumliche Verteilung der Austauschprozesse für die drei berechneten Szenarien

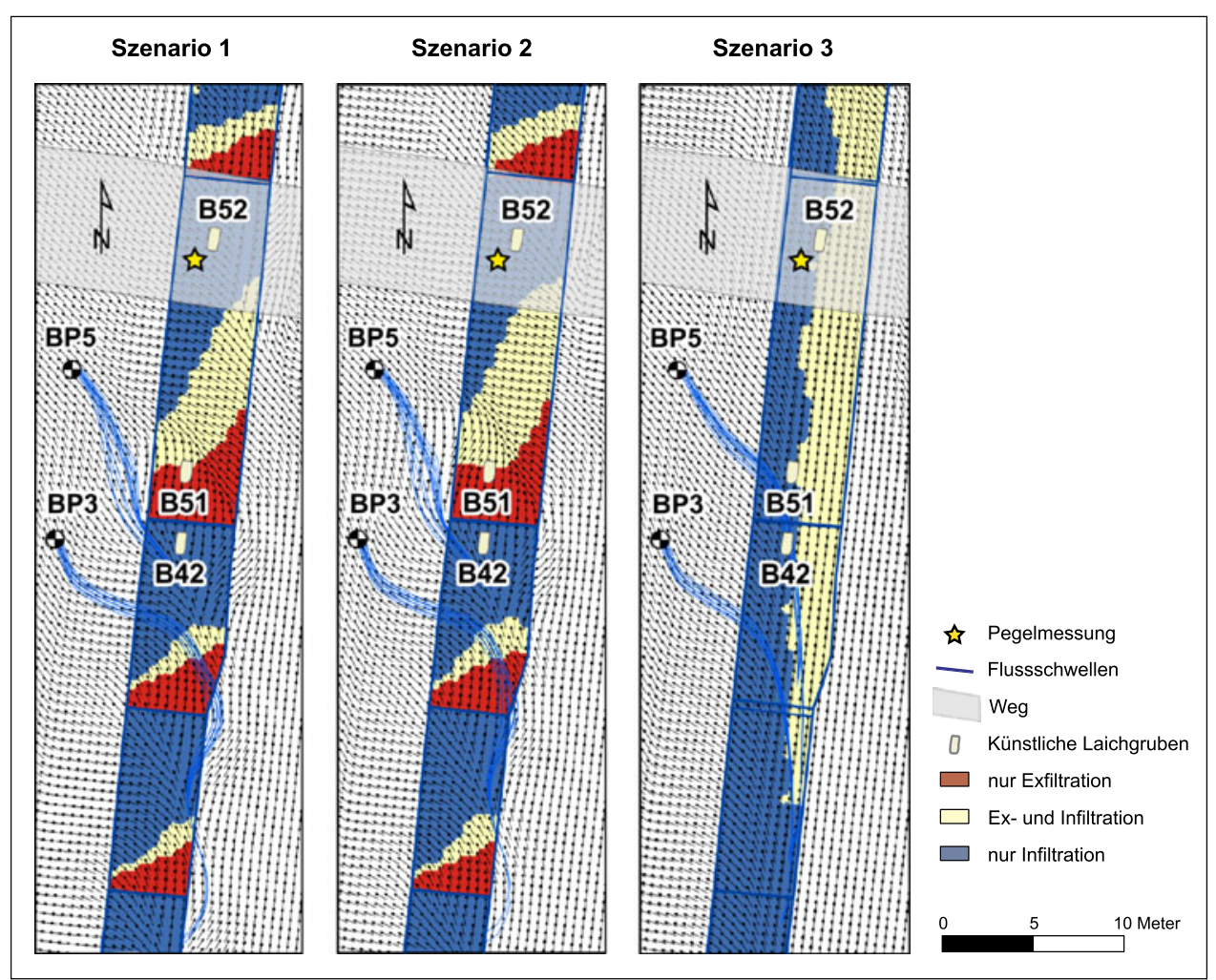

2 leicht ab. Die Berechnungen für Szenario 3 zeigen einen sehr ausgeglichenen Verlauf mit ausschließlich Infiltration von Flusswasser in das hyporheische Interstitial. Infiltrationsmengen liegen unter $0,5 \mathrm{~m}^{2} / \mathrm{d}$. Austauschprozesse werden vom regionalen Grundwasserfließregime und bevorzugter Infiltration von Flusswasser in das hyporheische Interstitial dominiert. Ein Ansteigen der Austauschraten am unteren Ende des untersuchten Flussabschnittes ist modelltechnisch begründet.

Abbildung 4 zeigt die zeitliche und räumliche Verteilung von In- und Exfiltration in den künstlich angelegten Forellenlaichgruben und entlang des untersuchten Flussabschnittes für die drei berechneten Szenarien. Auch hier bestehen für die Szenarien 1 und 2 kaum Unterschiede. Berechnete Austauschraten durch die Forellenlaichgruben sind für Szenario 3 stark reduziert und ein häufiger Wechsel von Inund Exfiltration kann beobachtet werden. Für die Szenarien 1 und 2 dominiert vor der Sohlschwelle die Infiltration von Flusswasser in das hyporheische Interstitial. Hinter der Sohlschwelle hingegen dominiert die Exfiltration von Grundwasser in das Flusswasser. Dazwischen existieren Zonen, in denen, in Abhängigkeit der Lage des Flusspegels, In- und Exfiltration abwechseln können. Die Berechnungen für Szenario 3 hingegen zeigen, dass vor allem zum linken Ufer hin, die Infiltration von Flusswasser in den Grundwasserleiter zunimmt. Zum rechten Ufer können In- und Exfiltration abwechseln. Ausschließlich Exfiltration, wie in den Szenarien 1 und 2, kann nicht mehr beobachtet werden. Al- le drei Szenarien zeigen, dass der Einfluss des regionalen Grundwasserfließregimes und Infiltrationsprozesse zum linken Ufer hin ansteigen.

Abbildung 5 veranschaulicht auch das Grundwasserfließregime für die verschiedenen Szenarien anhand von Fließvektoren und Zuströmbereichen zu den verschiedenen Grundwassermessstellen. Der Einfluss der Sohlschwellen ist für die Szenarien 1 und 2 deutlich zu erkennen. Interessant ist der Zuströmbereich zu Grundwassermessstelle BP3; Flusswasser infiltriert oberhalb einer Sohlschwelle in das hyporheische Interstitial und fließt unterhalb des Flussbettes der Grundwassermessstelle BP3 zu. Das Grundwasserfließfeld für Szenario 3 ist hingegen, wie zu erwarten, sehr viel gleichmäßiger.

Tabelle 4 fasst die berechneten Aufenthaltszeiten sowie die Länge der Fließpfade von Flussinfiltrat zwischen der Enziwigger und den verschiedenen Grundwassermessstellen zusammen. Für das lokale Modell und alle drei Szenarien benötigt infiltriertes Flusswasser für einen Fließpfad von ca. $90 \mathrm{~m}$ ca. 14 Tage um Grundwassermessstelle BP1 im Tal zu erreichen. Im ufernahen Bereich unterscheiden sich Länge der Fließpfade und Fließzeiten hingegen für die ersten beiden und das letzte Szenario. Für die beiden ersten Szenarien benötigt infiltriertes Flusswasser für Fließpfade von ca. 13 und $21 \mathrm{~m}$ Fließzeiten von ca. 4,2 und 4,5 Tage um die ufernahen Grundwassermessstellen zu erreichen. Für das letzte Szenario hingegen erreicht infiltriertes Flusswasser die ufer- 
nahe Grundwassermessstelle schon nach ca. 3,2 und 3,3 Tagen und Fließpfaden von ca. 22 und $24 \mathrm{~m}$.

\section{Diskussion}

Die Ergebnisse der Datenanalysen und der Grundwassermodellierung zeigen die große zeitliche und räumliche Variabilität der Austauschraten zwischen dem Oberflächengewässer und dem Grundwasser in Abhängigkeit der lokalen und regionalen Grundwasserfließregime. Diese Prozesse wiederum verändern die Strömungsdynamik, die Anteile an Wasserkomponenten unterschiedlicher Herkunft sowie die Wassertemperatur im hyporheischen Interstitial und im Bereich der Laichgruben. Die verschiedenen Faktoren, welche einen unterschiedlich starken Einfluss auf die Prozesse der Fluss-Grundwasser-Interaktion haben, werden nachfolgend anhand der Untersuchungsergebnisse diskutiert.

Niederschlags- und Schneeschmelzereignisse im Zusammenhang mit dem Verlauf der Lufttemperatur können indirekt über die Abflussbildung im Fließgewässer und lokal über oberflächige und unterirdische Hangzuflüsse einen starken Einfluss darstellen. Dies wird durch Zeitreihenanalysen der meteorologischen Daten bestätigt, welche zeigen, dass Hochwasser mit Niederschlags- oder Schneeschmelzereignissen korrelieren. Die Grundwasserneubildung durch flächig versickerndes Niederschlagswasser und der direkte Einfluss auf das regionale Grundwasserfließregime sind gering ( $<1 \%$ der Gesamtwasserbilanz). Ober- und unterirdische Hangzuflüsse haben einen mittleren Einfluss auf die Flussdynamik und lokale Fließregime in der hyporheischen Zone. Berechnete In- und Exfiltrationsraten zeigen, dass Variationen der In- und Exfiltration vor allem auf den Verlauf des Flusspegels zurückzuführen sind (Abb. 3). Die Exfiltration von Grundwasser in das Fließgewässer ist mengenmäBig im Vergleich zum Abflussvolumen klein.

Im Untersuchungsgebiet der Enziwigger führt die Geometrie des Grundwasserleiters sowie der starke Gradient vom Fluss zum Niveau des regionalen Grundwasserstroms dazu, dass auf der regionalen Skala der Prozess der Flusswasserinfiltration dominiert (Abb. 1, 2 und Tab. 1, 3). Auf der lokalen Skala im ufernahen Bereich und im Bereich der Sohlschwellen hingegen wechseln in- und exfiltrierende Verhältnisse in rascher Abfolge. Wichtigste Parameter für den Wechsel von In- und Exfiltration sind das Verhältnis von fluss- zum ufernahen Grundwasserpegel, die Durchlässigkeit der Flusssohle sowie die Flussbettmorphologie (Abb. 3, 5). Die Datenanalysen von Grundwasserpegel und -temperaturen zeigen, dass das regionale Grundwasserregime zeitverzögert das Signal aus Hochwasserereignissen widerspiegelt (Abb. 2 und Tab. 3). Das Signal aus Hochwasserereignissen wird in der hyporheischen Zone gedämpft. Dabei dominieren lokal in Bereichen oberhalb von
Sohlschwellen infiltrierende und in Bereichen unterhalb von Sohlschwellen exfiltrierende Verhältnisse. Weiterhin kann eine thermische Retardation zwischen dem Verlauf der Luftund der Grundwassertemperaturen beobachtet werden.

Vergleicht man die Resultate der verschiedenen hydraulischen und physikalischen Parameter, so lassen sich folgende Aussagen ableiten: (A) Eine Auswertung der Fluss- und Grundwasserpegeldaten zeigt wie die Druckfortpflanzung während Hochwasserereignissen stattfindet; dabei muss kein eigentlicher Massentransport von Flusswasser in den Grundwasserleiter stattfinden; (B) die Analyse der Fortpflanzung von Temperaturverläufen vom Oberflächengewässer in den Grundwasserleiter kann durch konduktiven Wärmetransport dominiert werden, wobei auch kein eigentlicher mit der Infiltrationsströmung verbundener advektiver Massentransport stattfinden muss; und (C) Änderungen der elektrischen Leitfähigkeiten im Grundwasser deuten auf echten Massentransport im hyporheischen Interstitial sowie Grundwasserleiter hin.

Mit den hochaufgelösten Grundwasserströmungsmodellen konnte das lokale und regionale Grundwasserfließregime beschrieben und Wasserbilanzen für die verschiedenen Untersuchungsskalen abgeleitet werden. In Abhängigkeit der betrachteten Skala machen Prozesse der In- und Exfiltration von Fluss- und Grundwasser $70 \%$ und $12 \%$ im regionalen sowie $86 \%$ und $30 \%$ im lokalen Maßstab an der Gesamtwasserbilanz aus. Der fehlende Anteil an der Gesamtwasserbilanz kann vor allem dem Hauptgrundwasserstrom im Tal zugewiesen werden. Der Einfluss, welcher die Instationarität des Grundwasserfließregimes ausmacht, kann auf die zeitliche und räumliche Verteilung der In- und Exfiltrationsprozesse zurückgeführt werden. Gleichzeitig konnte mit dem lokalen Modell die räumliche und zeitliche Instationarität von Austauschraten untersucht werden. Die mit den Modellen berechneten Fließgeschwindigkeiten liegen im ufernahen Bereich bei ca. 3 bis $5 \mathrm{~m} / \mathrm{d}$ und vom Fluss zum Talgrundwasserleiter bei ca. 6 bis $7 \mathrm{~m} / \mathrm{d}$. Die größeren Fließgeschwindigkeiten im Bereich des Talgrundwasserleiters sind auf den starken hydraulischen Gradienten vom Fluss zur Talmitte zurückzuführen. Die berechneten Fließgeschwindigkeiten sind in der gleichen Größenordnung wie Untersuchungsresultate aus vergleichbaren Grundwasserleitern (Hoehn \& von Gunten 1989).

Vor den Sohlschwellen nimmt die Infiltration von Flusswasser in das hyporheische Interstitial stark zu. Unterhalb der Schwellen findet eine Exfiltration von Grundwasser in den Fluss statt. Mit dem Grundwassermodell berechnete Austauschraten durch das Flussbett liegen im Bereich von $-0,24$ bis $1,35 \mathrm{~m} / \mathrm{d}$. Die aus den Temperaturanalysen in den künstlichen Forellenlaichgruben abgeleiteten Austauschraten liegen im Bereich von 0,11 bis $0,13 \mathrm{~m} / \mathrm{d}$. Sowohl die mit dem Modell als auch mit der Temperaturanalyse berechneten Austauschraten sind mit erhobenen Werten in an- 
deren Schweizer Fließgewässern vergleichbar (Hoehn 2002, Huggenberger et al. 2006).

Die Szenarienberechnungen erlaubten es, die Austauschraten und Fließgeschwindigkeiten des Modells mit hochaufgelöster vermessener Flussbetttopographie mit den Modellszenarien mit monotonerer Flussbetttopographie zu vergleichen. Alle drei Szenarien zeigen den Einfluss des regionalen Grundwasserfließregimes und die Zunahme der Infiltrationsprozesse zum linken Ufer hin. Für die ersten beiden Szenarien ist der Unterschied der räumlichen und zeitlichen Verteilung von Austauschraten vernachlässigbar. Die Ergebnisse lassen sich wie folgt interpretieren: Szenario 1 beschreibt die aktuelle Situation mit heterogenen Flussbettstrukturen, einschließlich gebildeten Kiesbänken und Kolkerscheinungen unterhalb der Sohlschwellen. Szenario 2 hingegen beschreibt die Situation eines stark verbauten Flusses kurz nach der Fertigstellung von flussbaulichen Maßnahmen. Die Modellresultate deuten also darauf hin, dass vor allem die ,harten Verbauungen“ (Sohlschwellen) im Flussbett die Austauschraten maßgeblich beeinflussen. Kleinskaligere Heterogenitäten spielen hingegen eher für die Wasserzirkulation im hyporheischen Interstitial der Kiesbänke eine Rolle. Szenario 3 beschreibt die Situation eines monotonen Flussbettverlaufs.

Die Ergebnisse der Detailuntersuchungen in den künstlich angelegten Forellenlaichgruben werden in Schindler Wildhaber et al. (in Vorbereitung) behandelt. Zusammenfassend können die folgenden Aussagen gemacht werden: (1) Die Sauerstoffmessungen in den künstlich angelegten Forellenlaichgruben B51 (Laichperiode 2009/2010 und 2010/2011) und B52 (Laichperiode 2010/2011) zeigen, dass während eines Großteils der Untersuchungsperioden für die Eientwicklung ausreichend Sauerstoff ( $>7 \mathrm{mg} / \mathrm{l}$; Crisp 2000) vorhanden war. Erst während des letzten Drittels vor Schlupf sanken die Werte zeitweise unter $7 \mathrm{mg} / \mathrm{l}$; (2) der Anteil von überlebenden Fischeiern liegt bei B52 bei $17 \%$ und bei B42 bei $27 \%$. Die Laichgrube B51 wurde in der Laichperiode 2009/2010 während eines Hochwasserereignisses im Dezember komplett weggeschwemmt. Während beider untersuchten Laichperioden wurden keine kritischen Temperaturen erreicht, welche die Fischeierentwicklung negativ beeinflusst hätten. Auch die modellierte hohe Variabilität der lokalen Grundwasserfließregime im hyporheischen Interstitial sowie die im Feld beobachtete eher geringe Beeinflussung der Flussbettdurchlässigkeit deuten auf einen positiven Einfluss bei der Eientwicklung hin. Trotzdem war das Überleben der Eier in der Enziwigger eher mäßig bis gering.

\section{Schlussfolgerungen}

Die Resultate der Untersuchungen zeigten, dass Grundwasserfließregime in einzelnen Flussabschnitten und im Bereich von einzelnen Laichgruben nicht isoliert von übergeordneten hydrologischen Randbedingungen betrachtet werden können. Der zeitlich und räumlich stark instationäre Charakter der Fluss-Grundwasser-Interaktion in den verschiedenen Skalen (regionales Grundwasserfließregime; Einfluss Sohlschwellen; Forellenlaichgruben) kann nur erfasst werden, indem man die regionalen und lokalen hydrologischen Randbedingungen mit Grundwasserströmungsmodellen simuliert.

Das regionale Grundwasserfließregime hat einen starken Einfluss auf die räumliche Verteilung der Austauschraten an der Gewässersohle der Enziwigger. Vor allem bedingt durch die Sohlschwellen im Fluss entwickeln sich lokale Grundwasserfließregime im hyporheischen Interstitial, welche in einer kleinräumigen Verteilung von Up- und DownwellingZonen resultieren. Oberhalb der Flussschwellen infiltriert Flusswasser aufgrund des erhöhten hydraulischen Gradienten. Das infiltrierte Wasser strömt um und unter der Schwelle und exfiltriert unterhalb der Schwelle wieder in das Fließgewässer. Diese lokalen Prozesse werden überlagert durch die instationären Prozesse der In- und Exfiltration zwischen dem Fließgewässer und dem regionalen Grundwasserfließregime.

Die Abflussdynamik hat in verbauten Fließgewässern wie der Enziwigger einen vergleichbar geringen Einfluss auf die Flussmorphologie und Flussbettdurchlässigkeit, umgekehrt ist der Einfluss der Flussmorphologie und Flussbettdurchlässigkeit auf die Strömungsdynamik vergleichsweise groß. Die Abflussdynamik hat einen starken Einfluss auf das lokale Fließregime in der hyporheischen Zone. Die Grundwasserzirkulation im hyporheischen Interstitial hängt $u$. a. von der Geometrie des Grundwasserleiters, dem Oberflächenabfluss, bzw. der Abflusstiefe, der Durchlässigkeit der Flusssohle und der Flussbettmorphologie ab. Eine Evaluation von Szenarien erlaubte eine Beurteilung, inwiefern zusätzliche Information des Flussbettes Modellresultate beeinflussen und welche Auflösung des Flussbettes für das Prozessstudium der Fluss-Grundwasser-Interaktion notwendig und relevant ist.

Danksagung Die Untersuchungen fanden in Rahmen des Schweizer Nationalfondsprojekts (K-32K1-120486) statt. Die Autoren wollen sich bei Daniel Altdorff und seinen Kollegen vom UFZ und Christian Michel für die gemeinsam durchgeführte Feldarbeit bedanken.

\section{Literatur}

Ashmore, P.E.: Bed-load transport in braided gravel-bed stream models. Earth Surf. Process. Landf. 13(8), 677-695 (1988)

Crisp, D.T.: Trout and Salmon: Ecology, Conservation and Rehabilitation. Fishing News Books, S. 224. Blackwell Sci., Oxford (2000)

Debele, B., Srinivasan, R.: Comparison of the performances of temperature-index and processbased energy budget snowmelt estimation approaches in the SWAT model, $40 \mathrm{~S}$. Texas A\&M University, Spatial Sciences Laboratory, College Station, TX, (2005) 
Doherty, J.: PEST model-independent parameter estimation. In: Watermark Computing, Corinda, Australia, 224 S. (1994)

Doppler, T., Franssen, H.J.H., Kaiser, H.P., Kuhlman, U., Stauffer, F.: Field evidence of a dynamic leakage coefficient for modelling river-aquifer interactions. J. Hydrol. 347(1-2), 177-187 (2007)

Environmental Modeling Systems I GMS: Groundwater Modeling System, South Jordan, Utah (2002)

Hatch, C.E., Fisher, A.T., Revenaugh, J.S., Constantz, J., Ruehl, C.: Quantifying surface water-groundwater interactions using time series analysis of streambed thermal records: method development. Wat. Resour. Res. 42(10), W10410 (2006)

Hoehn, E.: Hydrogeological issues of riverbank filtration-a review. In: Ray, C. (Hrsg.) Riverbank Filtration: Understanding Contaminant Biogeochemistry and Pathogen Removal, S. 17-41. Kluver Academic, Dordrecht (2002)

Hoehn, E., Cirpka, O.A.: Assessing residence times of hyporheic ground water in two alluvial flood plains of the Southern Alps using water temperature and tracers. Hydrol. Earth Syst. Sci. 10(4), 553-563 (2006)

Hoehn, E., von Gunten, H.R.: Radon in groundwater-a tool to assess infiltration from surface waters to aquifers. Water Resour. Res. 25(8), 1795-1803 (1989)

Huggenberger, P., Epting, J., Spottke, I., Regli, C., Zechner, E.: INTERREG III: A-Projekt MoNit „Modellierung der Grundwasserbelastung durch Nitrat im Oberrheingraben“", $110 \mathrm{~S}$. Landesanstalt für Umwelt, Messungen und Naturschutz Baden-Württemberg. Teilprojekte Nitratherkunft (Nitrat-Transportmodellierung) und
Fluss-Grundwasser-Interaktion (regionale hydrologische Grundlagendaten), (2006)

Keery, J., Binley, A., Crook, N., Smith, J.W.N.: Temporal and spatial variability of groundwater-surface water fluxes: development and application of an analytical method using temperature time series. J. Hydrol. 336(1-2), 1-16 (2007)

Landeshydrologie und -geologie: Geologischer Atlas der Schweiz (1994)

McDonald, M.G., Harbaugh, A.W.: Programmer's documentation for MODFLOW-96, an update to the U.S. geological survey modular finite-difference ground-water flow model, U.S. Geological Survey open-file report, S. 96-486 (1996)

Naegeli, M.W., Hartmann, U., Meyer, E.I., Uehlinger, U.: POMdynamics and community respiration in the sediments of a floodprone prealpine river (Necker, Switzerland). Arch. Hydrobiol. 133, 339-347 (1995)

Schager, E., Peter, A.: Bachforellensömmerlinge: Phase I. Netzwerk Fischrückgang Schweiz 315 (2001)

Schager, E., Peter, A.: Bachforellensömmerlinge: Phase II. Netzwerk Fischrückgang Schweiz 218 (2002)

Schälchli, U.: Die Kolmation von Fliessgewässersohlen: Prozesse und Berechnungsgrundlagen, Bd. 124. ETH, Zürich (1993)

Schälchli, U.: Basic equations for siltation of riverbeds. J. Hydraul. Eng. 121(3), 274-287 (1995)

Schindler Wildhaber, Y., et al.: Effects of sediment deposition on hydrology, oxygen supply and salmonid embryo in a channelized small river. Sci. Total Environ. (2013, in Vorbereitung) 